\title{
Cardiac Spiral Wave Termination by Linear Regional Cooling Toward the Anatomical Boundary of the Heart
}

\author{
Hiroshi Seno ${ }^{1} \cdot$ Naoki Tomii $^{2} \cdot$ Masatoshi Yamazaki $^{1} \cdot$ Haruo Honjo $^{3} \cdot$ Nitaro Shibata $^{4} \cdot$ Ichiro Sakuma $^{1}$
}

Received: 14 November 2019 / Accepted: 10 March 2020 / Published online: 18 March 2020

(c) The Author(s) 2020

\begin{abstract}
Purpose We hypothesized that linear regional cooling (LRC) toward the atrio-ventricular groove (AV-G) can move the spiral wave (SW) center to the AV-G effectively and terminate SW. The effectiveness of LRC in ex vivo 2D ventricle rabbit experiments was tested.

Methods We developed an experimental system to operate LRC and optical mapping simultaneously. To realize simultaneous cooling and optical mapping, a transparent cooling device was developed. LRC for $60 \mathrm{~s}$ toward 2D subepicardial ventricular myocardium of Langendorff-perfused rabbit hearts $(n=4)$ was conducted during constant pacing and persistent ventricular tachyarrhythmias (VTs).

Results Action potential duration at $90 \%$ repolarization $\left(\mathrm{APD}_{90}\right)$ at the cooling area was prolonged by LRC from 187 to $228 \mathrm{~ms} .41 \%$ of persistent VTs were terminated by LRC (12/29 cases). Cases where the original SW center moved toward the AV-G were observed via optical mapping. However, there were some cases where VT was not terminated by LRC. When the action potential duration (APD) of VT sustained cases were analyzed, LRC prolonged APD, but the APD prolonged area did not move toward the AV-G in most VT sustained cases

Conclusion Proper LRC toward the AV-G near the original SW center could move this center toward the AV-G and terminate SW excitation.
\end{abstract}

Keywords Arrhythmia $\cdot$ Ventricular tachycardia $\cdot$ Optical mapping $\cdot$ Regional cooling $\cdot$ Defibrillation

\section{Introduction}

Abnormal rapid heartbeat is called tachyarrhythmia. Theoretical and experimental studies have revealed that spiral wave (SW) excitation plays an important role to sustain tachyarrhtyhias $[1,2]$. Surgical cardiac ablation is often operated for radical cure of tachyarrhythmias. To realize accurate radical treatment, ablation strategies and mapping systems

H. Seno and N. Tomii contributed equally to this paper.

Hiroshi Seno

seno@bmpe.t.u-tokyo.ac.jp

1 Graduate School of Engineering, The University of Tokyo, Tokyo, Japan

2 Graduate School of Medicine, The University of Tokyo, Tokyo, Japan

3 Research Institute of Environmental Medicine, Nagoya University, Nagoya, Japan

4 Shinjuku Mitsui Building Clinic, Tokyo, Japan of cardiac excitation are being considered [3-7]. Recently, a patient-tailored computer simulation model was proposed to determine the appropriate ablation site for each patient [8]. However, cardiac ablation is an irreversible procedure, and only non-paroxysmal patients can undergo cardiac ablation and can be treated. On the other hand, in order to treat lethal arrhythmias such as ventricular tachycardia (VT) and ventricular fibrillation (VF) symptomatically, defibrillation by electric shock is often used. However, some adverse effects also exist with this treatment such as myocardial damages [9], increase in pacing threshold [10], mechanical dysfunction [11], increased risk of sudden cardiac death [12], and mental disorders [13]. Therefore, low-energy defibrillation is required.

For low-energy defibrillation, various methods have been proposed. The first approach was optimization of the shock waveform and energy, which was achieved by using biphasic shock [14, 15]. Another approach was using a combination of low-energy pacing pulses. Antitachycardia pacing is one of these approaches and is now clinically 
approved. This method is, however, known to be effective in terminating slow tachycardias, but the effectiveness against fast tachycardias and fibrillation was limited [16]. Recently, low-voltage stimulus was proposed to terminate SW excitation [17-22]. Rantner et al. proved that their low-voltage defibrillation protocol could terminate SW excitation with less electric power than conventional defibrillation methods [23]. Although they showed the possibility of treatment without pain, their study is still at the level of computer simulation experiments and the effectiveness in vivo remains unclear. In another study, DC shock with less electric power during regional cooling (RC) was proposed [24]. In this study, RC significantly decreased the defibrillation energy threshold. Moreover, it is reported that only RC without electrical shock terminated SW excitation in some cases. The mechanism of VT termination only by RC was also discussed, and the study concluded that RC destabilized the SW center to move along the periphery of the cooled region, which caused subsequent collision with boundaries of the heart called the atrio-ventricular groove (AV-G), which would, in turn, terminate SW excitation. From this study, it is possible that proper RC could terminate SW excitation without failure, and this method could be less-invasive defibrillation without electrical shock.

In this paper, to make SW termination by RC more reliable, we propose linear regional cooling (LRC), which cools the heart from SW center to the boundary of the heart. The assumed mechanism of SW termination by LRC is as follows: SW center would move around the periphery of LRC, move toward the AV-G of the heart effectively, and finally terminate (Fig. 1). To verify this hypothesis, we constructed a novel LRC system for rabbit hearts, conducted LRC toward the VT-induced rabbit hearts, and simultaneously measured the membrane potential distribution by optical mapping.
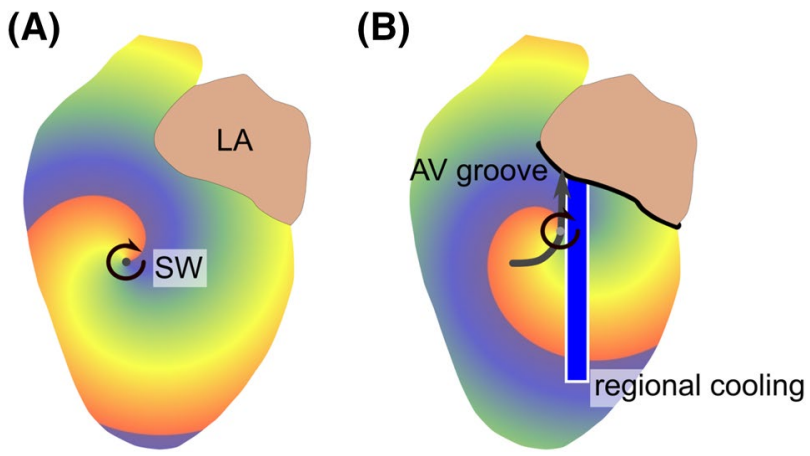

Fig. 1 Schematic diagram of spiral wave (SW) termination by linear regional cooling. a Initial state of SW excitation. b After applying linear regional cooling toward the $\mathrm{AV}$ groove. $R V$ right ventricular, $L V$ left ventricular, $L A$ left atrium, $S W$ spiral wave, $A V$ groove atrioventricular groove

\section{Method}

We established an experimental system to perform RC of epicardial myocardium and simultaneously measured the membrane potential by optical mapping. Figure 2a shows the schema of the experimental system. In brief, a cooling device with a liquid channel was attached to the heart, and epicardial cooling was performed with the cooling device where coolant was flown through the fabricated channel. Simultaneously, optical mapping was carried out with an excitation light-emitting diode (LED) and high-speed camera.

\subsection{Cooling System}

Detailed design of the cooling device and a schematic diagram of the experimental design are shown in Fig. 2b, c. For assessing simultaneous cooling and optical mapping, a transparent cooling device with a fluid channel was made with polydimethylsiloxane (PDMS) and polyethylene film. The fluid channel was $50 \mathrm{~mm}$ in height, $9 \mathrm{~mm}$ in width, and $9 \mathrm{~mm}$ in depth. For enhanced cooling efficiency between the coolant and heart, a $30 \mathrm{~mm} \times 3 \mathrm{~mm}$ hole was bored in the channel, and the hole was sealed with polyethylene (thickness: approximately $11 \mu \mathrm{m}$ ) as a cooling window. Figure $2 \mathrm{~d}$ shows the camera view for optical measurement. For better cooling efficiency and optical measurement quality, a 50\% ethylene glycol aqueous solution (freezing point: approximately $-30{ }^{\circ} \mathrm{C}$, specific heat: approximately $0.75 \mathrm{cal} / \mathrm{g} /{ }^{\circ} \mathrm{C}$ at $0{ }^{\circ} \mathrm{C}$ ) was used as a coolant.

To evaluate the cooling ability of this system, a preliminary phantom experiment was performed. Polyacrylamide gel was prepared as a phantom representation of heart tissue. This phantom contained micro capsuled thermochromic liquid crystal (MTLC, Japan capsule products) and thermocouple (ThreeHigh Co., Ltd) for temperature detection. The visualized temperature range of this MTLC was from 30 to $40{ }^{\circ} \mathrm{C}$. The thermocouple was located $1 \mathrm{~mm}$ below the cooling window.

\subsection{Experimental Model and the Optical Mapping System}

The ex vivo experiment's protocol was approved by the Institutional Animal Care and Use Committee of the University of Tokyo. Male Japanese white rabbits weighing $2.5-2.99 \mathrm{~kg}$ $(n=4)$ were used. The experimental models were essentially equivalent to previous studies [25]. The hearts were quickly removed from anesthetized rabbits, and the isolated hearts were perfused on a Langendorff apparatus with Krebs-Ringer solution. A complete atrio-ventricular block 
(A)

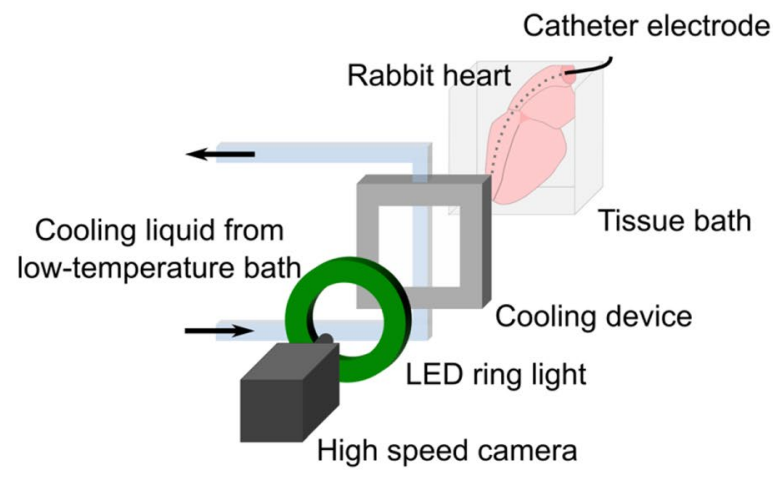

(B)

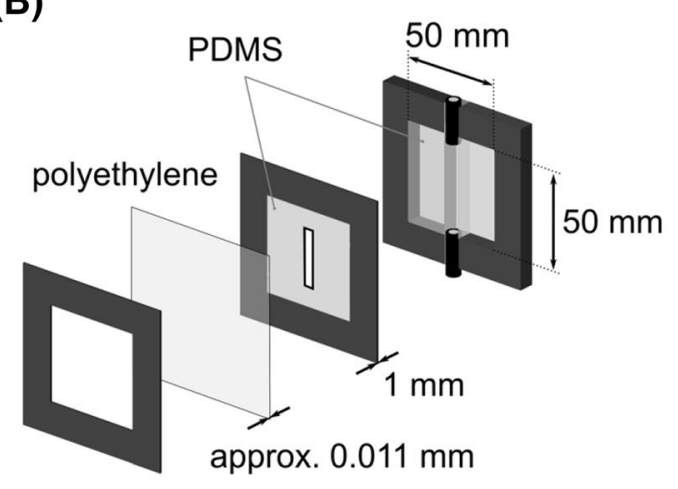

(C)

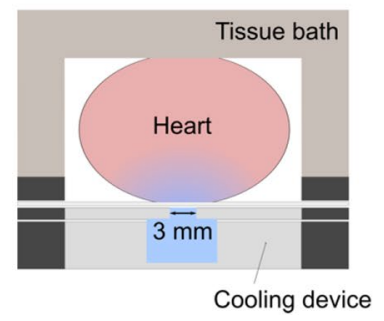

(D)

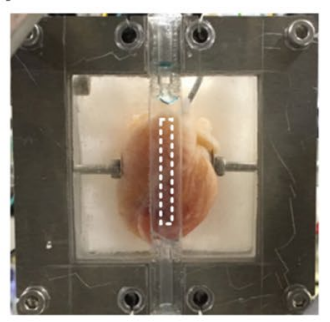

(E)

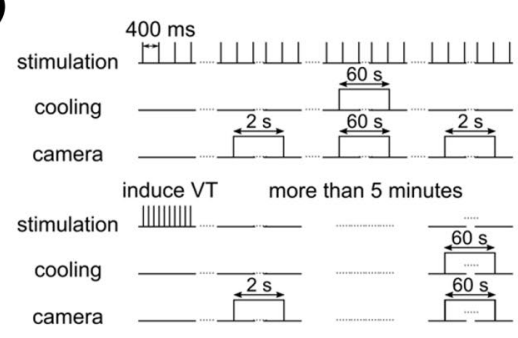

Fig. 2 Schema of the linear cooling system for rabbit heart and the experimental protocol. a The regional cooling system for rabbit heart was developed. An extracted rabbit heart was fixed in a tissue bath, which has a transparent cooling device as a front wall. Low-temperature liquid was stored in the low-temperature bath, and low-temperature liquid was sent to the cooling device by changing the fluid path. Membrane potentials were recorded by a fluorescent measurement called optical mapping. b Basic design for the cooling device. This cooling device was fabricated with polydimethylsiloxane (PDMS) and polyethylene film for florescent signals to pass through. The cooling device had a fluid path (height: $50 \mathrm{~mm}$, width: $9 \mathrm{~mm}$, depth: $9 \mathrm{~mm}$ ). For enhanced heat transfer, a cooling window (height: $30 \mathrm{~mm}$, width: $3 \mathrm{~mm}$, depth: $1 \mathrm{~mm}$ ) where low-temperature liquid could exchange the heat with the heart through polyethylene film (thickness: approximately $11 \mu \mathrm{m}$ ) was prepared. This transparent

was made by ligation and ablation of the atrio-ventricular node. The Langendorff-perfused rabbit heart was immersed in a tissue bath containing perfusion fluid at $37^{\circ} \mathrm{C}$, and after inserting a copper hand-made cryoprobe in the left ventricular cavity, the probe was filled with liquid nitrogen at $-192{ }^{\circ} \mathrm{C}$ for $5 \mathrm{~min}$ to ablate endocardial myocardium. These hearts were dyed with Di-4-ANEPPS $(0.2 \mu \mathrm{M})$ for the fluorescent measurement of membrane potential called optical mapping [26]. In addition, $15 \mathrm{mM}$ 2,3-butanedione monoxime (BDM) was applied as an electro-contractile uncoupler to minimize motion artifacts from the heart.

Di-4-ANEPPS was used as a voltage-sensitive dye with $520 \mathrm{~nm}$ excitation wavelength. For excitation light, LED (primary wavelength: $530 \mathrm{~nm}$, LEX2-LZ4-G, Brainvision Inc., Japan) was used. High-pass optical filter (passing $>600$ nm, R-60, Nikon, Japan) was used to record only cooling device was stabilized using an aluminum frame. c Top view of the cooling device during the experiment. Low temperature liquid could effectively absorb heat from the rabbit heart through polyethylene film. d Actual picture of the designed cooling device. The white dotted square in this picture indicates the location of the cooling window. The cooling device was attached to an extracted heart. e Timing chart for stimulation, regional cooling, and optical measurement during constant pacing and ventricular tachycardia (VT). Constant stimulations were applied from bipolar electrodes located in the endocardium of the right ventricle at the cycle length of $400 \mathrm{~ms}$. Membrane potentials were recorded before, during, and after linear regional cooling. VT was induced by burst pacing from the same electrode as the above experiment. Membrane potentials before regional cooling were measure first. Linear regional cooling was done for persistent VT, which continues for $>5 \mathrm{~min}$

fluorescent signals, and the fluorescent light that passed through was recorded by a high-speed camera (500 fps, 256 pixel $\times 256$ pixel, FASTCAM SA-4, Photron, Japan).

\subsection{Experimental Protocol}

LRC and optical mapping were simultaneously performed during constant pacing and persistent VTs. Figure 2e represents the timing chart for each experiment.

To evaluate the cooling effect toward the rabbit heart, LRC during constant pacing was assessed. Stimulation was applied from a bipolar electrode located in the endocardium of the right ventricle. The stimulus intensity was twice as large as the diastolic threshold at a pacing cycle length of $400 \mathrm{~ms}$. As a control measurement, membrane potential distribution without LRC was observed for $2 \mathrm{~s}$. Then, LRC 
and optical mapping were simultaneously performed. As a result of the preliminary phantom experiment, coolant kept at $-15^{\circ} \mathrm{C}$ was used. The period of cooling was set to $60 \mathrm{~s}$ not to damage the heart tissue by severe cooling. More than 5 min after LRC, membrane potential distribution in the rewarmed heart was observed. The change in action potential duration (APD) was analyzed.

LRC during persistent VTs was also executed. VT was induced by burst pacing from the same bipolar electrode as above. First, optical mapping was immediately performed after VT induction. If VT lasted for more than $5 \mathrm{~min}$, this excitation was considered to be persistent VT. Then, LRC and optical mapping were executed. The period and temperature of coolant were the same as the constant pacing experiment. Termination of persistent VT was judged from endocardium electrode signals and/or optical signals.

\subsection{Analysis}

To evaluate the cooling effect on the rabbit heart, APD was calculated. First, times of depolarization and repolarization were identified from normalized membrane potentials. Then the time interval between these two times was defined as APD. APD at $90 \%$ repolarization $\left(\mathrm{APD}_{90}\right)$ and $\mathrm{APD}$ at $50 \%$ repolarization $\left(\mathrm{APD}_{50}\right)$ were calculated.

Phase analysis has been proposed to analyze SW center during VTs [27]. Moreover, to detect spatiotemporal movement of SW center during VTs, phase variance analysis has been proposed [28]. Taking these two, both the phase map and the phase variance map were calculated from the membrane potential distribution to analyze the SW center movement during VTs. Briefly, the details of the process are as follows: First, phase value was calculated from membrane potential signals at each pixel by applying the Hilbert transform function. Second, the variances of phase around each pixel were calculated and mapped on the phase variance map. The window size for evaluation of the phase variance was 9 pixels $\times 9$ pixels, and the value of the phase variance ranged from 0 to 1 . A high phase variance area (phase variance value $>0.8$ ) was defined as the SW center.

\section{Results}

\subsection{Evaluation of Established Cooling System}

Figure $3 \mathrm{a}, \mathrm{b}$ show the temperature change detected by thermocouple and MTLC in a preliminary phantom experiment. From Fig. 3a, the temperature of the heart phantom decreased faster when lower temperature coolant was used. When coolant kept at $-15{ }^{\circ} \mathrm{C}$ was used, the temperature decreased by $3{ }^{\circ} \mathrm{C}$ within the first $20 \mathrm{~s}$, then $10^{\circ} \mathrm{C}$ within the next $40 \mathrm{~s}$. Figure $3 \mathrm{~b}$ shows the temperature distribution measured by MTLC. This result shows that the cooling region had a width of $\sim 8 \mathrm{~mm}$.

Figure $3 \mathrm{c}, \mathrm{d}$ indicate the spatial pattern of $\mathrm{APD}_{90}$ and the average of $\mathrm{APD}_{90}$ obtained from ex vivo experiment. As visible in Fig. $3 c, \mathrm{APD}_{90}$ regionally prolonged along the cooling channel. From Fig. 3d, the average $\mathrm{APD}_{90}$ was $\sim 187 \mathrm{~ms}$ before LRC. After starting LRC, $\mathrm{APD}_{90}$ in the cooling window extended with time and reached $\sim 228 \mathrm{~ms}$ after $60 \mathrm{~s}$ cooling. Furthermore, $\mathrm{APD}_{90}$ shortened to $191 \mathrm{~ms}$ after rewarming the heart.

\subsection{Cooling Against Persistent Ventricular Tachyarrhythmias}

Overall, persistent VTs were induced 29 times. Among them, LRC terminated about $41 \%$ of the VTs one minute (12/29 cases). The average time for termination of VTs was $26.7 \pm 15.2 \mathrm{~s}$. Figure $4 \mathrm{a}-\mathrm{c}$ shows typical SW excitation when persistent VT terminated. Figure $4 \mathrm{a}$ includes three images, which represent the location of the SW center and wave front superimposed on the raw camera image. In these images, clockwise SW was observed. In the second image $(t=20.784 \mathrm{~s})$, the SW center drastically moved upward. When the wave front propagated and reentered the cooling region, this SW center moved upward again and terminated $(t=20.788 \mathrm{~s})$. Figure $4 \mathrm{~b}$ indicates the isochronal map of 20.6-20.8 s after cooling. In this map, the functional block line (black line) toward AV-G was observed. Figure $4 \mathrm{c}$ shows the membrane potential signals at 4 points across the functional block line (shown in Fig. 4b). Points 1 and 2 are located on the left side of the functional block line, and points 3 and 4 were on the right side of the functional block line. In this figure, membrane potentials at points 1 and 2 were depolarized, whereas excitation did not propagate through points 3 and 4 . Therefore, conduction block occurred around the functional block line within this period. However, there were unsuccessful cases where LRC for $60 \mathrm{~s}$ could not terminate SW excitation. Figure 4d includes images that illustrate the SW center and wave front. In these images, new SW centers appeared when the wave front collided with cooling region $(t=59.774 \mathrm{~s})$. However, these SW centers were annihilated by collision with each other $(\mathrm{t}=59.788 \mathrm{~s})$. Then, SW excitation was sustained. Figure $4 \mathrm{e}, \mathrm{f}$ show the isochronal map and membrane potential signals around the functional block line. From the isochronal map, there was an isolated functional block line, and the wave front was broken into two around this region. Moreover, depolarization was weakened in this region (Fig. 4f). Therefore, the conduction blockage was thought to have occurred around the cooling region.

To analyze why LRC could not terminate persistent VT, the $\mathrm{APD}_{50}$ at $60 \mathrm{~s}$ after LRC was assessed. Four cases whose signal-to-noise ratio of the membrane potential 
Fig. 3 Evaluation of the cooling ability of the established cooling system. a Temperature measurements during the preliminary phantom cooling experiment. Temperature was measured by a thermocouple fixed in polyacrylamide phantom $1 \mathrm{~mm}$ below the cooing window. The temperature was decreased faster when lowertemperature liquid was used as coolant. When ethylene glycol aqueous solution kept at $-15^{\circ} \mathrm{C}$ was used, the temperature was decreased by about $3{ }^{\circ} \mathrm{C}$ for $20 \mathrm{~s}$ then $10{ }^{\circ} \mathrm{C}$ for $1 \mathrm{~min}$. b Temperature measurement during the phantom experiment by micro capsuled thermochromic liquid crystal (MTLC). The tissue phantom was cooled with approximately $8 \mathrm{~mm}$ width. c Spatial distribution of the action potential duration at $90 \%$ repolarization $\left(\mathrm{APD}_{90}\right)$ of the extracted rabbit heart. $\mathrm{APD}_{90}$ prolonged along the cooling region. d Spatial average of $\mathrm{APD}_{90}$ inside the cooling window (white dotted square in the camera image). The average $\mathrm{APD}_{90}$ inside the cooling window reached about $228 \mathrm{~ms}$ after $60 \mathrm{~s}$, whereas the average $\mathrm{APD}_{90}$ before cooling and after rewarming the heart was $187 \mathrm{~ms}$ and $191 \mathrm{~ms}$, respectively
(A)

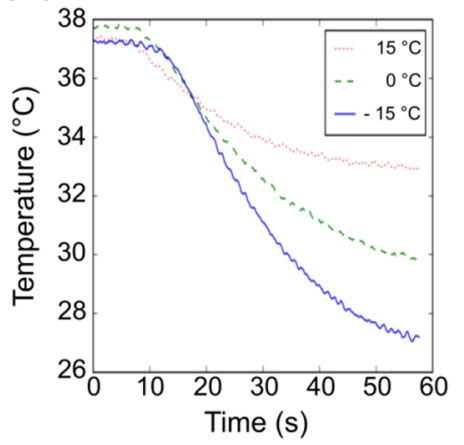

(B)
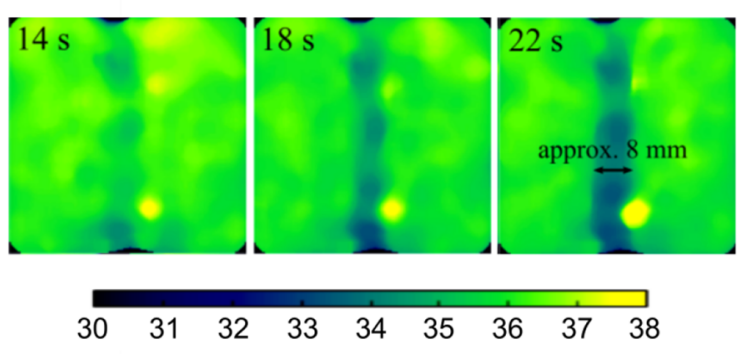

Temperature $\left({ }^{\circ} \mathrm{C}\right)$

(C)

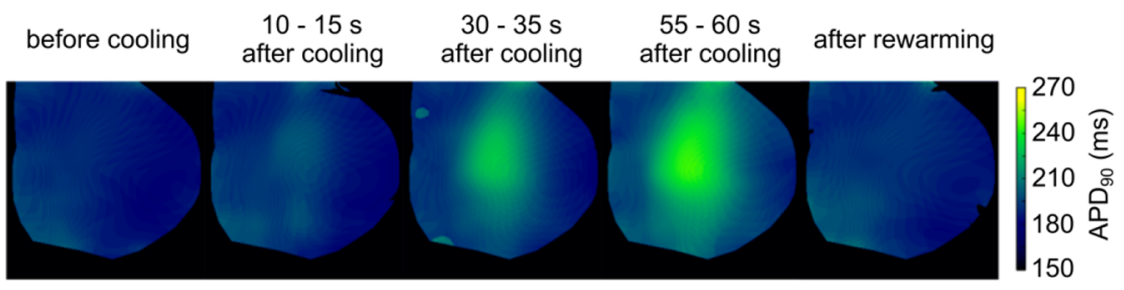

(D)

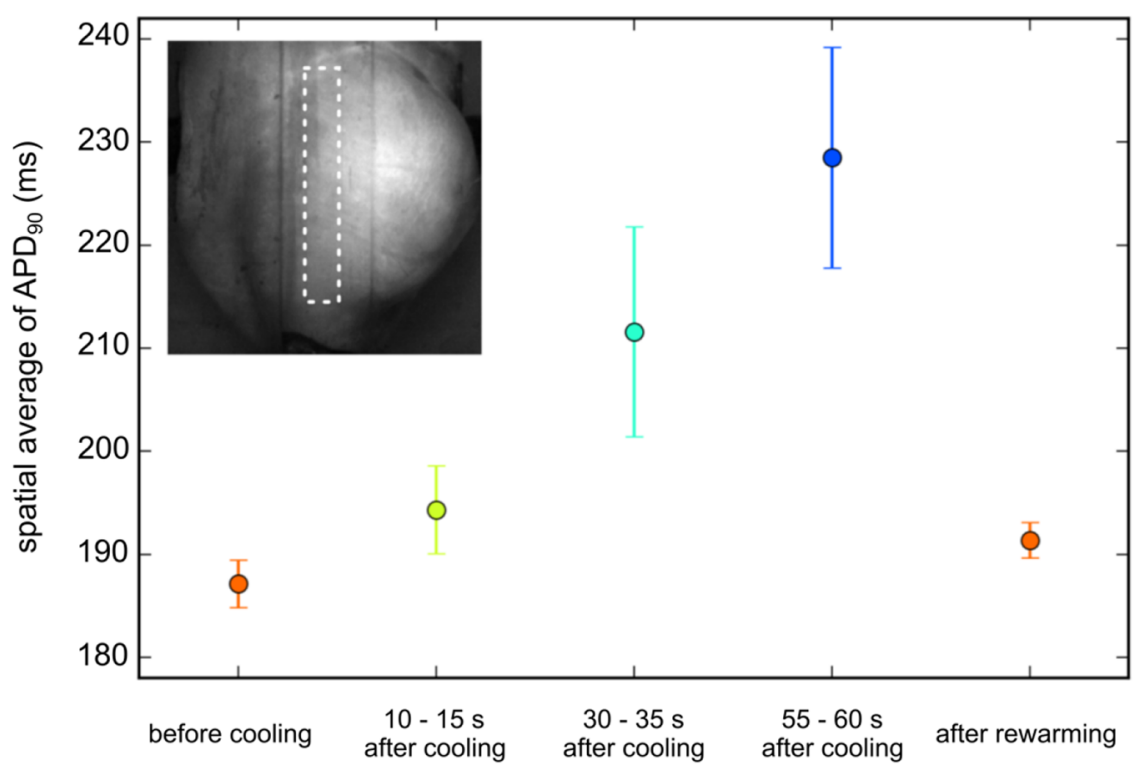

signals was poor were excluded from 17 VT sustained cases. Figure 5 indicates four typical cases. Figure 5 a shows the $\mathrm{APD}_{50}$ distribution when VT was terminated. In this case, $\mathrm{APD}_{50}$ was prolonged, and the prolonged area was connected to the AV-G. On the other hand, Fig. 5b-d indicates the $\mathrm{APD}_{50}$ distribution when VT sustained. In Fig. 5b, $\mathrm{APD}_{50}$ appears to have been prolonged, and the prolonged APD region was connected to the AV-G. However, in this case, VT was sustained. Figure 5c shows that the $\mathrm{APD}_{50}$ was also prolonged, but the $\mathrm{APD}_{50}$ prolonged area was not connected to the AV-G. In Fig. 5d, APD ${ }_{50}$ was not prolonged even after LRC. The ratio of each unsuccessful case was $7.7 \%(1 / 13), 61.5 \%(8 / 13)$, and $30.8 \%$ (4/13).

\section{Discussion}

The LRC system in rabbit heart was established, and a phantom experiment as well as ex vivo cooling experiment were conducted. In the phantom experiment, the established LRC system could cool the phantom at a certain speed $\left(10^{\circ} \mathrm{C}\right.$ temperature decrease for $60 \mathrm{~s}$ ) and locality ( $8 \mathrm{~mm}$ width), as shown in Fig. 3a, b. Ex vivo experiments were conducted using extracted rabbit hearts $(n=4)$. From analyzing the membrane potentials during constant pacing, the cooling system could prolong $\mathrm{APD}_{90}$ from 187 to $228 \mathrm{~ms}$ in $60 \mathrm{~s}$ cooling (Fig. 3c, d). In addition, the cooling system could terminate $41 \%$ of persistent VTs (12/29), which were sustained for more than 5 min. The time until VT termination was $26.7 \pm 15.2 \mathrm{~s}$. The analysis 
(A)

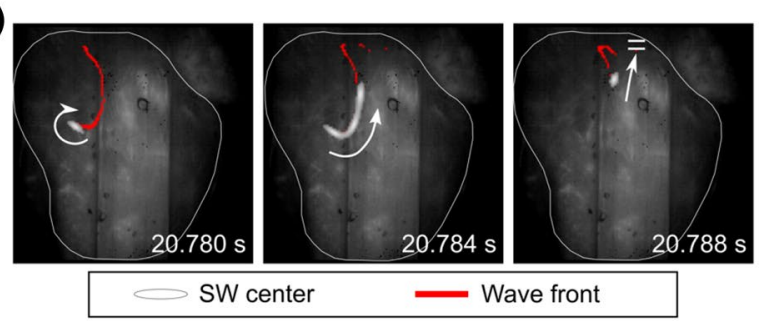

(B)

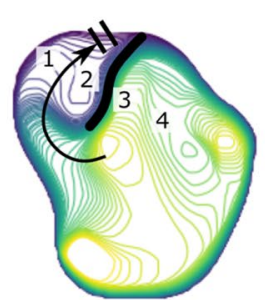

(D)
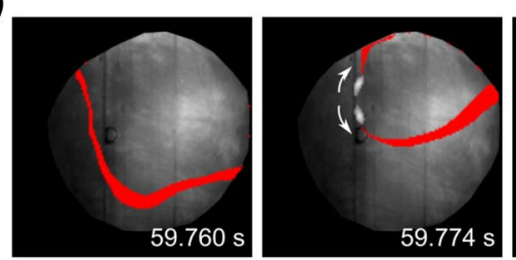

(C)

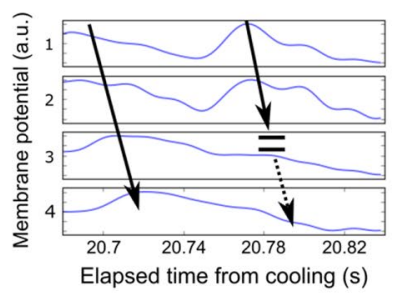

(E)
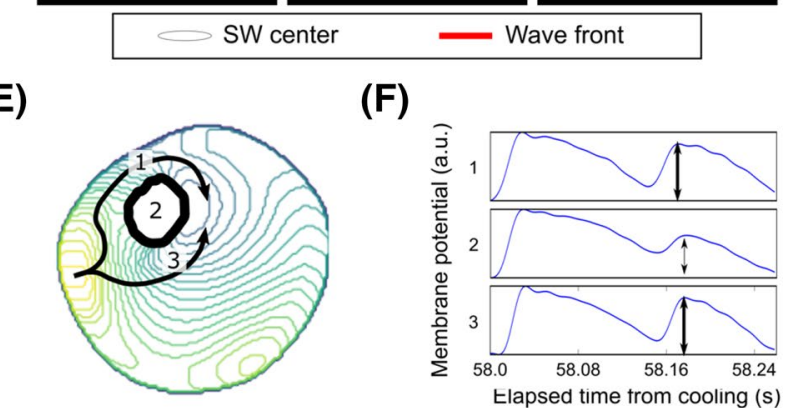

(F)

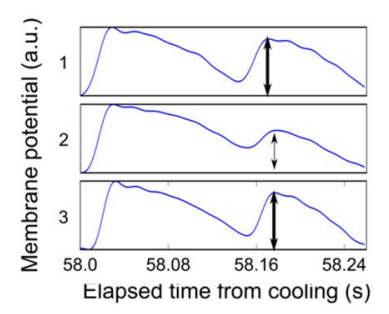

Fig. 4 Typical example of spiral wave (SW) excitation during linear regional cooling (LRC). a Movement of the SW center (white area) and wave front (red line) superimposed on the raw camera image when VT was terminated $\sim 21 \mathrm{~s}$ after LRC. The SW center moved along the LRC and toward the top of the heart where the atrio-ventricular groove is located as the anatomical block line. b Isochronal map when the SW center moved toward the anatomical block line. The functional block line (black line) was observed near the atrioventricular groove. c Membrane potential signals at 4 points around the functional block line. The excitation at points 3 and 4 did not occur, and this means conduction blockage occurred. $\mathbf{d}$ Movement of the SW center (white area) and wave front (red line) superimposed on the raw camera image when VT was not terminated $60 \mathrm{~s}$ after LRC. New SW centers were generated around the cooling region, but these two SWs collided with each other and were annihilated. e Isochronal map when new SW centers were generated and collided. There was an isolated functional block line, and the wave was broken into two around the region. f Membrane potential signals at three points around the functional block line. Depolarization of the membrane potential was weakened inside the functional block line

of membrane potential distribution during LRC showed that the SW center moved along the cooling region, and persistent VTs were terminated in some cases as hypothesized
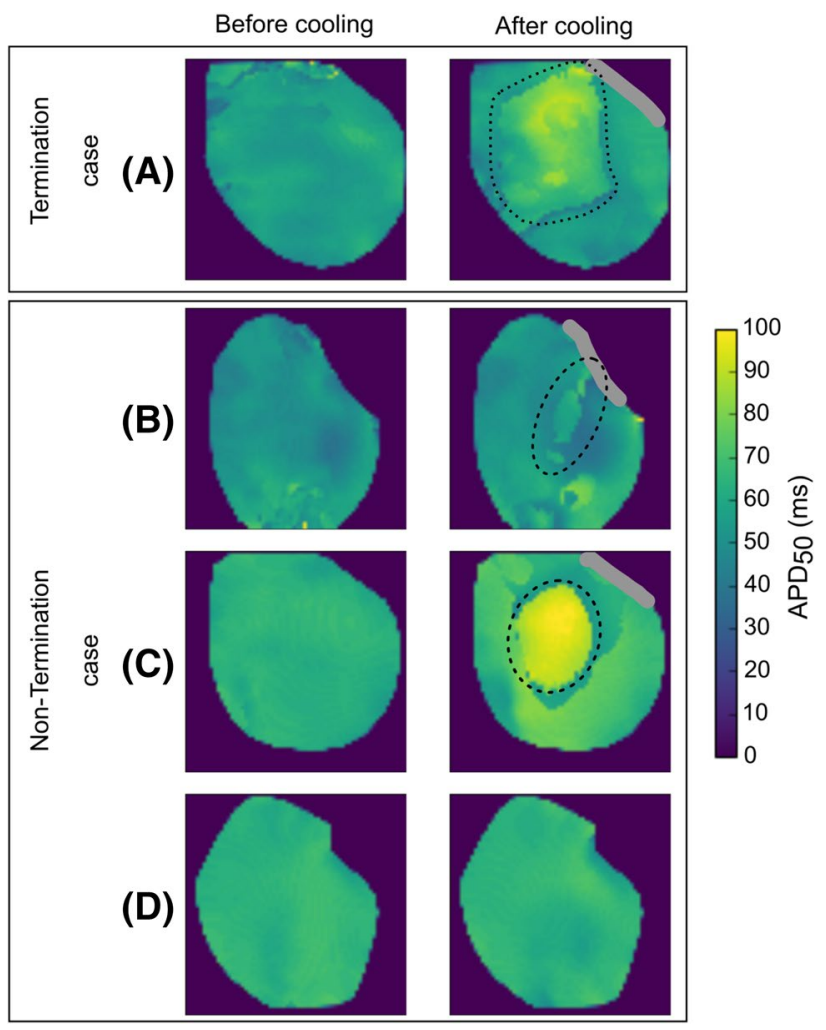

Fig. 5 Comparison of the action potential duration at 50\% repolarization $\left(\mathrm{APD}_{50}\right)$ before and after LRC. The gray line represents the atrioventricular groove. a Transition of $\mathrm{APD}_{50}$ when LRC could terminate persistent VT. $\mathrm{APD}_{50}$ was prolonged, and the prolonged region was connected to the atrio-ventricular groove. b Typical example of APD transition when persistent VT sustained. APD was prolonged, and the prolonged region was connected to the atrio-ventricular groove. Therefore, ideal LRC could be accomplished, but LRC could not terminate VT in this case. The ratio of this transition pattern was $7.7 \%$ (1/13). c Other examples of APD transition with persistent sustained VT. $\mathrm{APD}_{50}$ was prolonged, but the prolonged region was not connected to the atrio-ventricular groove. The ratio of this transition pattern was $61.5 \%(8 / 13)$. d Another example of APD transition when persistent VT was sustained. APD was not prolonged even after $60 \mathrm{~s}$ of LRC. The ratio of this transition pattern was 30.8\% (4/13)

(Fig. 4a-c). However, there were some cases where LRC for $60 \mathrm{~s}$ could not terminate persistent VTs. For example, in 1 case, conduction block occurred, but the original SW was conserved (Fig. 4d-f). Analysis of the $\mathrm{APD}_{50}$ during VT showed that the $\mathrm{APD}_{50}$ prolonged region was connected to the AV-G in VT terminated cases, whereas the $\mathrm{APD}_{50}$ prolonged area not connected to AV-G or $\mathrm{APD}_{50}$ was not prolonged in most VT sustained cases (Fig. 5).

\subsection{Termination Rate of VTs}

In an ex vivo experiment, $\sim 41 \%$ of VTs $(12 / 29)$ could be terminated by LRC and the rest, 59\% (17/29) could not be terminated by LRC for $60 \mathrm{~s}$. 
One possible reason why LRC could not terminate VT is the initial location of the SW. In our experiment, the original SW center was located outside of the region of optical mapping in many cases because the view was restricted, which means that the original SW center was located far from the cooling region. If the SW center moves along the cooling region observed in successful cases (Fig. 4a-c), the SW center should be located near the cooling region to be terminated. Indeed, there was a case where APD was prolonged, and the APD prolonged region was connected to the AV-G, but persistent VT was sustained (Fig. 5b). Perhaps VT in this case could not be terminated because the original SW was far from the cooling region.

Another possibility is the relation between the cooled region and the AV-G. When the $\mathrm{APD}_{50}$ during VT was analyzed, the APD prolonged region was not connected to the AV-G in $61.5 \%$ (8/13) of VT sustained cases (Fig. 5c). Moreover, there was a case where LRC did not prolong APD (Fig. 5d). This means that LRC toward AV-G was not conducted properly. This may be because the cooling device was not completely attached to the heart and a softer cooling device should be developed to cool the heart surface more effectively. Therefore, to move the SW center toward AV-G and terminate VT, LRC should be connected to the AV-G as initially hypothesized (Fig. 1).

In summary, LRC toward AV-G near the SW center was appropriate for terminating VT. Only one cooling line was set to terminate VTs because the objective was to elucidate the VT termination mechanism by LRC and the region for optical measurement was limited. Therefore, the termination rate for VTs could be improved by optimizing the cooling region for the whole heart.

\subsection{Mechanism of SW Drift Along the Cooling Region}

The reason why RC can induce conduction blocks and move the SW center to the AV-G can be discussed based on cardiac electrophysiology.

In general, cooling of cardiac tissue prolongs the APD, which was observed in our ex vivo experiment (Fig. 3c, d). Considering the effect of cooling on the SW dynamics, APD prolongation is the prolongation of the refractory period, and cooling aids the generation of conduction blocks. Moreover, cardiac cell membranes consist of plethoric ion channels and pumps, and each ion channel and pump is temperaturesensitive [29-33]. From previous research, the conductance of the sodium channel is also affected by temperature [34]. This means that propagation of excitation, that is mainly characterized by sodium channel dynamics, can be weakened by cooling. From this point of view, it is a reasonable assumption that conduction blocks occurred around the cooling region.
To discuss SW dynamics after conduction blockage, occurrence and annihilation dynamics of SWs should be considered. In a previous study about point stimulation during SW excitation, SW dynamics after conduction block were also discussed [35]. The study reported that conduction block generates two SWs, one clockwise and another counterclockwise. Both SWs annihilate when clockwise SW collides with counterclockwise SW. Considering the SW dynamics, the mechanism of SW drift during RC is thought to be the following: First, cooling prolongs APD and weakens the propagation of excitation, which generates conduction blocks. The conduction block generates two SWs. One of them collides with the original SW and annihilates it. Finally, the other SW sustains, and the original SW center moves along the cooling region.

\subsection{Study Limitations}

We used the 2D ventricular myocardium of a rabbit heart and confirmed that proper LRC can terminate SW excitation. However, it is well-known that excitation in 3D cardiac tissue can be more complex. Therefore, it is essential to evaluate the effects of LRC in $3 \mathrm{D}$ cardiac tissue. Moreover, only VT was induced in these experiments. This is because we considered the simple excitation pattern of VT is appropriate for verifying the principle of SW termination by LRC. Future experiments will elucidate the termination mechanism of more complex excitation like VF with LRC. Our proposed method can be used as the defibrillator without electrical shock. However, there are many difficulties to be solved to apply the method to human heart: cooling device should be directly attached to the heart and coolant or power supplier for a cooling element should be kept in the body, which may cause problems about safety and energy efficiency. Due to the difficulties, clinical cooling device does not exist as far as we know, and such devices have to be developed to apply our method to the human heart in a clinical situation.

\section{Conclusion}

We established a RC system in rabbit hearts and verified our hypothesis that LRC toward the AV-G can move the SW center effectively and terminate SW excitation. It was shown that LRC could terminate SW excitation when LRC toward the AV-G was properly conducted and the cooling region was close to the original SW center. This study suggests that blocking conduction near the SW center toward the AV-G could be beneficial for the termination of reentrant activity like VT and VF. 
Acknowledgements This study was supported by a Grant-in Aid for Scientific Research 18K18357 to Dr. Tomii, 18H02802 to Dr. Yamazaki, and 15H01801 and 18H04161 to Dr. Sakuma, from the Japan Society for Promotion of Science.

Open Access This article is licensed under a Creative Commons Attribution 4.0 International License, which permits use, sharing, adaptation, distribution and reproduction in any medium or format, as long as you give appropriate credit to the original author(s) and the source, provide a link to the Creative Commons licence, and indicate if changes were made. The images or other third party material in this article are included in the article's Creative Commons licence, unless indicated otherwise in a credit line to the material. If material is not included in the article's Creative Commons licence and your intended use is not permitted by statutory regulation or exceeds the permitted use, you will need to obtain permission directly from the copyright holder. To view a copy of this licence, visit http://creativecommons.org/licenses/by/4.0/.

\section{References}

1. Jalife, J. (2000). Ventricular fibrillation: Mechanisms of initiation and maintenance. Annual Review of Physiology, 62(1), 25-50. https://doi.org/10.1146/annurev.physiol.62.1.25.

2. Weiss, J. N., Qu, Z., Chen, P.-S., Lin, S.-F., Karagueuzian, H. S., Hayashi, H., et al. (2005). The dynamics of cardiac fibrillation. Circulation, 112(8), 1232-1240. https://doi.org/10.1161/CIRCU LATIONAHA.104.529545.

3. Nademanee, K., McKenzie, J., Kosar, E., Schwab, M., Sunsaneewitayakul, B., Vasavakul, T., et al. (2004). A new approach for catheter ablation of atrial fibrillation: mapping of the electrophysiologic substrate. Journal of the American College of Cardiology, 43(11), 2044-2053. https://doi.org/10.1016/j.jacc.2003.12.054.

4. Haissaguerre, M., Hocini, M., Denis, A., Shah, A. J., Komatsu, Y., Yamashita, S., et al. (2014). Driver domains in persistent atrial fibrillation. Circulation, 130(7), 530-538. https://doi.org/10.1161/ CIRCULATIONAHA.113.005421.

5. Seitz, J., Bars, C., Théodore, G., Beurtheret, S., Lellouche, N., Bremondy, M., et al. (2017). AF ablation guided by spatiotemporal electrogram dispersion without pulmonary vein isolation: A wholly patient-tailored approach. Journal of the American College of Cardiology, 69(3), 303-321. https://doi.org/10.1016/j. jacc.2016.10.065.

6. Baykaner, T., Rogers, A. J., Meckler, G. L., Zaman, J., Navara, R., Rodrigo, M., et al. (2018). Clinical implications of ablation of drivers for atrial fibrillation: A systematic review and metaanalysis. Circulation: Arrhythmia and Electrophysiology, 11(5), e006119. https://doi.org/10.1161/CIRCEP.117.006119.

7. Sakata, K., Okuyama, Y., Ozawa, T., Haraguchi, R., Nakazawa, K., Tsuchiya, T., et al. (2018). Not all rotors, effective ablation targets for nonparoxysmal atrial fibrillation, are included in areas suggested by conventional indirect indicators of atrial fibrillation drivers: ExTRa Mapping project. Journal of Arrhythmia, 34(2), 176-184. https://doi.org/10.1002/joa3.12036.

8. Boyle, P. M., Zghaib, T., Zahid, S., Ali, R. L., Deng, D., Franceschi, W. H., et al. (2019). Computationally guided personalized targeted ablation of persistent atrial fibrillation. Nature Biomedical Engineering. https://doi.org/10.1038/s41551-019-0437-9.

9. Al-Khadra, A., Nikolski, V., \& Efimov, I. R. (2000). The role of electroporation in defibrillation. Circulation Research, 87(9), 797-804. https://doi.org/10.1161/01.RES.87.9.797.

10. Weaver, W. D., Cobb, L. A., Copass, M. K., \& Hallstrom, A. P. (1982). Ventricular defibrillation-A comparative trial using
175-J and 320-J shocks. New England Journal of Medicine, 307(18), 1101-1106. https://doi.org/10.1056/NEJM19821028307 1801.

11. Waldecker, B., Brugada, P., Zehender, M., Stevenson, W., \& Wellens, H. J. J. (1986). Dysrhythmias after direct-current cardioversion. The American Journal of Cardiology, 57(1), 120-123. https://doi.org/10.1016/0002-9149(86)90963-X.

12. Tokano, T., Bach, D., Chang, J., Davis, J., Souza, J. J., Zivin, A., et al. (1998). Effect of ventricular shock strength on cardiac hemodynamics. Journal of Cardiovascular Electrophysiology, 9(8), 791-797. https://doi.org/10.1111/j.1540-8167.1998.tb001 18.x.

13. Larsen, G. K., Evans, J., Lambert, W. E., Chen, Y., \& Raitt, M. H. (2011). Shocks burden and increased mortality in implantable cardioverter-defibrillator patients. Heart Rhythm, 8(12), 1881-1886. https://doi.org/10.1016/J.HRTHM.2011.07.036.

14. Matula, M. H., Brooks, M. J., Pan, Q. I., Pless, B. D., Province, R. A., \& Echt, D. S. (1997). Biphasic waveforms for ventricular defibrillation: Optimization of total pulse and second phase durations. PACE - Pacing and Clinical Electrophysiology, 20(9 I), 2154-2162. https://doi.org/10.1111/j.1540-8159.1997.tb04231.x.

15. Mouchawar, G., Kroll, M., Val-Mejias, J. E., Schwartzman, D., McKenzie, J., Fitzgerald, D., et al. (2000). ICD waveform optimization: A randomized, prospective, pair-sampled multicenter study. PACE - Pacing and Clinical Electrophysiology, 23(11 II), 1992-1995. https://doi.org/10.1111/j.1540-8159.2000.tb07070.x.

16. Wathen, M. S., DeGroot, P. J., Sweeney, M. O., Stark, A. J., Otterness, M. F., Adkisson, W. O., et al. (2004). Prospective randomized multicenter trial of empirical antitachycardia pacing versus shocks for spontaneous rapid ventricular tachycardia in patients with implantable cardioverter-defibrillators: Pacing fast ventricular tachycardia reduces shock therapies (PainFREE Rx II) trial results. Circulation, 110(17), 2591-2596. https://doi. org/10.1161/01.CIR.0000145610.64014.E4.

17. Fenton, F. H., Luther, S., Cherry, E. M., Otani, N. F., Krinsky, V., Pumir, A., et al. (2009). Termination of atrial fibrillation using pulsed low-energy far-field stimulation. Circulation, 120(6), 467476. https://doi.org/10.1161/CIRCULATIONAHA.108.825091.

18. Luther, S., Fenton, F. H., Kornreich, B. G., Squires, A., Bittihn, P., Hornung, D., et al. (2011). Low-energy control of electrical turbulence in the heart. Nature, 475(7355), 235-239. https://doi. org/10.1038/nature10216.

19. Li, W., Ripplinger, C. M., Lou, Q., \& Efimov, I. R. (2009). Multiple monophasic shocks improve electrotherapy of ventricular tachycardia in a rabbit model of chronic infarction. Heart Rhythm, 6(7), 1020-1027. https://doi.org/10.1016/j.hrthm.2009.03.015.

20. Ambrosi, C. M., Ripplinger, C. M., Efimov, I. R., \& Fedorov, V. V. (2011). Termination of sustained atrial flutter and fibrillation using low-voltage multiple-shock therapy. Heart Rhythm, 8(1), 101-108. https://doi.org/10.1016/j.hrthm.2010.10.018.

21. Li, W., Janardhan, A. H., Fedorov, V. V., Sha, Q., Schuessler, R. B., \& Efimov, I. R. (2011). Low-energy multistage atrial defibrillation therapy terminates atrial fibrillation with less energy than a single shock. Circulation: Arrhythmia and Electrophysiology, 4(6), 917-925. https://doi.org/10.1161/CIRCEP.111.965830.

22. Janardhan, A. H., Li, W., Fedorov, V. V., Yeung, M., Wallendorf, M. J., Schuessler, R. B., et al. (2012). A novel low-energy electrotherapy that terminates ventricular tachycardia with lower energy than a biphasic shock when antitachycardia pacing fails. Journal of the American College of Cardiology, 60(23), 2393-2398. https ://doi.org/10.1016/j.jacc.2012.08.1001.

23. Rantner, L. J., Tice, B. M., \& Trayanova, N. A. (2013). Terminating ventricular tachyarrhythmias using far-field low-voltage stimuli: Mechanisms and delivery protocols. Heart Rhythm, 10(8), 1209-1217. https://doi.org/10.1016/j.hrthm.2013.04.027. 
24. Yamazaki, M., Honjo, H., Ashihara, T., Harada, M., Sakuma, I., Nakazawa, K., et al. (2012). Regional cooling facilitates termination of spiral-wave reentry through unpinning of rotors in rabbit hearts. Heart Rhythm: The Official Journal of the Heart Rhythm Society, 9(1), 107-114. https://doi.org/10.1016/j.hrthm .2011.08.013.

25. Yamazaki, M., Honjo, H., Nakagawa, H., Ishiguro, Y. S., Okuno, Y., Amino, M., et al. (2007). Mechanisms of destabilization and early termination of spiral wave reentry in the ventricle by a class III antiarrhythmic agent, nifekalant. American Journal of Physiology Heart and Circulatory Physiology, 292(1), H539-H548. https ://doi.org/10.1152/ajpheart.00640.2006.

26. Kodama, I., Honjo, H., Yamazaki, M., Nakagawa, H., Ishiguro, Y., Okuno, Y., et al. (2005). Optical imaging of spiral waves: Pharmacological modification of spiral-type excitations in a 2-dimensional layer of ventricular myocardium. Journal of Electrocardiology, 38(4), 126-130. https://doi.org/10.1016/J.JELEC TROCARD.2005.06.025.

27. Gray, R. A., Pertsov, A. M., \& Jalife, J. (1998). Spatial and temporal organization during cardiac fibrillation. Nature, 392(6671), 75-78. https://doi.org/10.1038/32164.

28. Tomii, N., Yamazaki, M., Arafune, T., Honjo, H., Shibata, N., \& Sakuma, I. (2016). Detection algorithm of phase singularity using phase variance analysis for epicardial optical mapping data. IEEE Transactions on Biomedical Engineering, 63(9), 1795-1803. https ://doi.org/10.1109/TBME.2015.2502726.

29. Cavalié, A., McDonald, T. F., Pelzer, D., \& Trautwein, W. (1985). Temperature-induced transitory and steady-state changes in the calcium current of guinea pig ventricular myocytes. Pflügers Archiv, 405(3), 294-296.
30. Kiyosue, T., Arita, M., Muramatsu, H., Spindler, A. J., \& Noble, D. (1993). Ionic mechanisms of action potential prolongation at low temperature in guinea-pig ventricular myocytes. The Journal of Physiology, 468, 85-106.

31. Nakamura, Y., Ohya, Y., Abe, I., \& Fujishima, M. (1999). Sodiumpotassium pump current in smooth muscle cells from mesenteric resistance arteries of the guinea-pig. The Journal of Physiology, 519(1), 203-212. https://doi.org/10.1111/j.1469-7793.1999.0203o .x.

32. Nagatomo, T., Fan, Z., Ye, B., Tonkovich, G. S., January, C. T., Kyle, J. W., et al. (1998). Temperature dependence of early and late currents in human cardiac wild-type and long Q-T $\triangle \mathrm{KPQ} \mathrm{Na}{ }^{+}$ channels. American Journal of Physiology-Heart and Circulatory Physiology, 275(6), H2016-H2024. https://doi.org/10.1152/ajphe art.1998.275.6.H2016.

33. Chen, Y., \& DeHaan, R. L. (1993). Temperature dependence of embryonic cardiac gap junction conductance and channel kinetics. Journal of Membrane Biology, 136(2), 125-134.

34. Milburn, T., Saint, D. A., \& Chung, S. H. (1995). The temperature dependence of conductance of the sodium channel: Implications for mechanisms of ion permeation. Receptors \& Channels, 3(3), 201-211.

35. Tomii, N., Yamazaki, M., Arafune, T., Kamiya, K., Nakazawa, K., Honjo, H., et al. (2018). Interaction of phase singularities on spiral wave tail: Reconsideration of capturing the excitable gap. American Journal of Physiology-Heart and Circulatory Physiology. https://doi.org/10.1152/ajpheart.00558.2017. 